VoL. 47 (1993) [523-524]

\title{
Asymptotic representation of transient electromagnetic fields in geophysical prospecting
}

\author{
RAINER IGNETIK
}

This thesis deals with the mathematical aspects of modelling problems arising in transient electromagnetic (TEM) prospecting, and in particular the asymptotic analysis of these fields in time and space. The problems appearing in geophysical prospecting for massive sulfide ore-bodies provide the main motivation for the thesis and lead to a wealth of interesting mathematical problems. The solutions for a loop in whole space are analysed in detail as generalised functions for Heaviside switch-off and several new switch-forms are considered. The late-time asymptotic representations of the field for impulsively initiated electromagnetic fields show that they are almost identical for a dipole and a finite current loop, within carefully specified ranges. The late-time asymptotic representation of the field within an inhomogeneous whole-space shows that TEM measurements can be confused with the response from layered medium.

The late-time asymptotic representations for the electric field on and above a permeable and conducting half-space is next developed for Heaviside switch-off and linear ramp switch-offs. The relevance of an impulsive start to the evolution of the electric field is discussed in detail in relation to the switch-off model. It is shown that the permeability dependence of the electric field can be expressed in terms of certain elementary functions rather than the more cumbersome hypergeometric functions. The electromagnetic field in a conducting and permeable ground is considered and in particular we consider the effect permeability has on the "smoke ring" propagation.

We derive the complete and explicit solutions for the electric field in the air for the problem of a sphere in a two-layered ground, allowing for permeability contrast between the various media. The analysis is carried to the stage where a Fourier inverse transform can be used to obtain the electric field in the time domain.

Two models for inhomogeneous conductivity profiles are studied. Specifically we consider a half-space with an exponentially decreasing and increasing profile with depth and derive the corresponding late-time asymptotic representation for the electric field.

Received 18th November, 1992

Thesis submitted to University College, University of New South Wales, Australian Defence Force Academy March 1992. Degree approved October, 1992. Supervisor: Associate Professor R.A. Sammut, Australian Defence Force Academy. Associate Supervisor: Professor K.C. Westfold, Monash University.

Copyright Clearance Centre, Inc. Serial-fee code: 0004-9729/93 \$A2.00+0.00. 
The exponentially decreasing model shows that it behaves like a slab in a resistive whole-space. The results are applicable to salinity mapping in Australia and can be extended to other conductivity profiles.

High Frequency Radar Division

Defence Science and Technology Organisation

PO Box 1500

Salisbury SA 5108 Australia 